\title{
EVALUATION OF TEST QUANTITIES FOR LEAKAGE DIAGNOSIS IN THE AIR PATH OF AN AUTOMOTIVE ENGINE
}

\author{
Mattias Nyberg \\ Department of Electrical Engineering, Linköping University, \\ Linköping, Sweden \\ Email: matny@isy.liu.se
}

\begin{abstract}
Two different methods for diagnosing leakage in the air-path of an automotive engine are investigated. The first is based on a comparison between measured and estimated air flows. The second is based on an estimation of the leakage area. The two methods are compared by using a framework of hypothesis testing and especially the power function. The investigation is made first in theory and then also on a real engine. The conclusion is that the principle based on the estimated leakage area, gives a better power function and is therefore the best choice if only leakage detection is considered. However, if also other faults need to be diagnosed, it is shown that the sensitivity to these other faults may be better with the principle based on comparison of estimated and measured air flow.
\end{abstract}

Keywords: Fault Diagnosis, Fault Isolation, Hypothesis Testing, Statistics

\section{INTRODUCTION}

One important area of automotive engine diagnosis is the diagnosis of leakage in the air-intake system. This is because a leakage can cause increased emissions, drivability problems, and damage to the engine. To diagnose leakages, test quantities (or residual generators) need to be constructed. It is important that these test quantities are as sensitive as possible to the leakage.

In this paper, a view of hypothesis testing is utilized. Then a natural performance measure for the hypothesis tests, and also the different test quantities, is the power function, i.e. the probability of rejecting the null-hypothesis when it is true. These concepts, in the context of fault diagnosis, are introduced in Section 2.

By the help of the power function, two different strategies for constructing test quantities are compared: one based on comparing estimated and measured air flow, and the other based on estimating the leakage area. The investigation is made first in theory, in Section 3, and then in Section 4 also on a real engine.

\section{MODEL BASED DIAGNOSIS IN THE VIEW OF HYPOTHESIS TESTING}

This section gives a short summary of the method structured hypothesis tests presented in (Nyberg, 1999b; Nyberg, 2000). First, we classify the different faults into fault modes. For example in an automotive engine, all intake-manifold leaks, regardless of their area, belong to the fault mode "manifold leak" which we will denote $M L$. Another fault mode is the case "no fault" which we will denote $N F$.

The exact fault of the process is described by the fault state $\theta$, which mostly is vector valued. The set of all possible fault states is denoted $\Theta$ and the different fault modes $\gamma$ divides $\Theta$ into disjunct subsets $\Theta_{\gamma}$.
For an example, consider a system described by the following equations:

$$
\begin{aligned}
\dot{x} & =f(x, u) \\
y_{1} & =h_{1}(x)+b_{1} \\
y_{2} & =h_{2}(x)+b_{2}
\end{aligned}
$$

The constants $b_{1}$ and $b_{2}$ represents sensor bias faults and it is assumed that only positive biases can occur. Three fault modes are considered: no fault $N F$, a bias in sensor $1 B 1$, and a bias in sensor $2 B 2$. The fault state of the system is described by the vector $\theta=\left[\begin{array}{ll}b_{1} & b_{2}\end{array}\right]$. Then we have the sets $\Theta=\left\{\left[b_{1} b_{2}\right] \mid b_{1} \geq\right.$ $\left.0, b_{2} \geq 0\right\}, \Theta_{N F}=\left\{\left[\begin{array}{ll}0 & 0\end{array}\right]\right\}, \Theta_{B 1}=\left\{\left[\begin{array}{ll}b_{1} & b_{2}\end{array}\right] \mid b_{1}>\right.$ $\left.0, b_{2}=0\right\}$, and $\Theta=\left\{\left[\begin{array}{ll}b_{1} & b_{2}\end{array}\right] \mid b_{1}=0, b_{2}>0\right\}$.

Using the principle of structured hypothesis tests, the diagnosis system consists of a set of hypothesis tests. The null hypothesis for the $k$ :th hypothesis test, i.e. $H_{k}^{0}$, is that the fault mode, present in the process, belongs to a specific set $M_{k}$ of fault modes. The alternative hypothesis $H_{k}^{1}$ is that the present fault mode does not belong to $M_{k}$. This means that if hypothesis $H_{k}^{0}$ is rejected, and thus $H_{k}^{1}$ is accepted, the present fault mode can not belong to $M_{k}$. In this way, each separate hypothesis test contributes with a piece of information about which fault modes that can be present.

For example, let $F_{p}$ denote the present fault mode and assume that the diagnosis system contains the following set of three hypothesis tests:

$\begin{array}{ll}H_{1}^{0}: F_{p} \in M_{1}=\left\{N F, F_{1}\right\} & H_{1}^{1}: F_{p} \in M_{1}^{C}=\left\{F_{2}, F_{3}\right\} \\ H_{2}^{0}: F_{p} \in M_{2}=\left\{N F, F_{2}\right\} & H_{2}^{1}: F_{p} \in M_{2}^{C}=\left\{F_{1}, F_{3}\right\} \\ H_{3}^{0}: F_{p} \in M_{3}=\left\{N F, F_{3}\right\} & H_{3}^{1}: F_{p} \in M_{3}^{C}=\left\{F_{1}, F_{2}\right\}\end{array}$

Then if only $H_{1}^{0}$ is rejected, we can draw the conclusion that $F_{p} \in M_{1}^{C}=\left\{F_{2}, F_{3}\right\}$, i.e. the present fault mode is either $F_{2}$ or $F_{3}$. If both $H_{1}^{0}$ and $H_{2}^{0}$ are 
rejected, we can draw the conclusion that $F_{p} \in M_{1}^{C} \cap$ $M_{2}^{C}=\left\{F_{2}, F_{3}\right\} \cap\left\{F_{1}, F_{3}\right\}=\left\{F_{3}\right\}$, i.e. the present fault mode is $F_{3}$.

From the above example, it is clear that the diagnosis, i.e. the output from the diagnosis system, is formed with a simple intersection operation. Note that the diagnosis statement can sometimes contain more than one fault mode. This feature corresponds well to a desired functionality in cases where it is difficult or even impossible to decide which fault mode has occurred.

\subsection{Hypothesis Tests}

For each hypothesis test $\mathrm{HT}_{k}$, we need to find a test quantity (often also called test statistic) and a rejection region. The sample data $\mathbf{x}$ for each hypothesis test are samples of process inputs $u$ and outputs $y$. The test quantity is a function $T_{k}(\mathbf{x})$ from the sample data $\mathbf{x}$, to a scalar value which is to be thresholded by a threshold $J_{k}$. Thus the rejection region is defined implicitly by the threshold $J_{k}$ together with the test quantity $T_{k}(\mathbf{x})$. The hypothesis test $\mathrm{HT}_{k}$ is then defined as

$$
\begin{array}{ll}
T_{k}(\mathbf{x})>J_{k} & \text { reject } H_{k}^{0}, \text { i.e. accept } H_{k}^{1} \\
T_{k}(\mathbf{x})<J_{k} & \text { do not reject } H_{k}^{0}
\end{array}
$$

This means that we need to design a test quantity $T_{k}(\mathbf{x})$ such that it is low if the data $\mathbf{x}$ match the hypothesis $H_{k}^{0}$, i.e. a fault mode in $M_{k}$ can explain the data. Also if the data come from a fault mode not in $M_{k}, T_{k}(\mathbf{x})$ should be high. These performance goals can be formalized by using the power function defined as

$$
\beta_{k}(\theta)=P\left(\text { reject } H_{k}^{0} \mid \theta\right)=P\left(T_{k}(x) \geq J_{k} \mid \theta\right)
$$

That is, we want the power function to be large for all $\theta \notin \Theta_{k}^{0}$, where $\left.\Theta_{k}^{0}=\cup_{\gamma \in M_{k}} \Theta_{\gamma}\right\}$.

\subsection{Principles for Constructing Test Quantities}

Test quantities can be calculated based on many different principles. In this paper we will consider two possibilities, here denoted "prediction principle" and "estimation principle".

2.2.1. Prediction Principle Using the prediction principle, the calculation of the test quantity is based on a model validity measure $V_{k}(\theta, \mathbf{x})$, which in turn is based on a comparison between signals and/or predictions (or estimates) of signals. The function $V_{k}(\theta, \mathbf{x})$, where $\theta$ is fixed, is a measure of the validity of the model of the system, for a fixed $\theta$, in respect to the measurement data $x$. To construct $V_{k}(\theta, \mathbf{x})$, we can compare an output signal $y$ with an estimate $\hat{y}$, but it is also possible to for example compare two estimates of the same signal. An example is

$$
V_{k}(\theta, x)=\frac{1}{N} \sum_{t=1}^{N}\|y(t)-\hat{y}(t \mid \theta, x)\|
$$

where $y(t \mid \theta, x)$ is the prediction of the output $y(t)$, derived from an assumption of a specific $\theta$ and the measured data $x$. The test quantity can then be calculated as

$$
T_{k}(x)=\min _{\theta \in \Theta_{k}^{0}} V_{k}(\theta, x)
$$

Note that the term "residual generator", as it is mostly used in fault diagnosis literature, refers in fact to a principle similar to the prediction principle.
2.2.2. Estimate Principle Using the estimate principle, the test quantity is based on a comparison between an estimate of $\theta_{i}$, i.e. an element $\theta_{i}$ of the fault state vector $\theta$, and the nominal value $\theta_{i}^{0}$. First consider the case where the set $\Theta_{k}^{0}$ consists of only one element. Then a test quantity can be constructed as

$$
T_{k}(\mathbf{x})=\left\|\hat{\theta}_{i}-\theta_{i}^{0}\right\| \quad \hat{\theta}_{i}=\arg \min _{\theta_{i}} V^{\prime}\left(\theta_{i}, \mathbf{x}\right)
$$

where $V^{\prime}\left(\theta_{i}, \mathbf{x}\right)$ is some model validity measure. This is a common solution used in literature, e.g. (Isermann, 1993). When the set $\Theta_{k}^{0}$ consists of more than one element, additional parameters have to be estimated. That is, in addition to estimating the parameter $\theta_{i}$, we also have to estimate the free parameters in $\Theta_{k}^{0}$, i.e. the ones corresponding to faults that are decoupled.

\section{A COMPARISON BETWEEN THE PREDICTION PRINCIPLE AND THE ESTIMATE PRINCIPLE}

Later in Section 4, we will compare the prediction principle with the estimate principle for diagnosing leakage in a real automotive engine. To support and explain the conclusions drawn there, we will in this section study some theoretical examples.

Consider a system which can be modeled as

$$
y(t)=b|u|^{\varphi} \operatorname{sgn} u+a+v
$$

where $v(t) \sim N\left(0, \sigma_{v}\right)$ and $\operatorname{sgn} u$ is the sign of $u$, i.e. $-1,0$, or 1 . The nominal (i.e. corresponding to the no fault case) values for the three parameters are $b_{0}=1$, $a_{0}=0$, and $\varphi_{0}=1$. The four fault modes considered are

$$
\begin{array}{ll}
N F & b=1, a=0, \varphi=1 \\
F_{b} & b \neq 1, a=0, \varphi=1 \\
F_{\varphi} & b=1, a=0, \varphi \neq 1 \\
F_{a} & b=1, a \neq 0, \varphi=1
\end{array}
$$

We will start by comparing two test quantities for the null hypothesis $H_{0}: F_{p}=N F$. The first, $T_{1}(x)$ is based on the prediction principle, and the second, $T_{2}(x)$ is based on the estimate principle:

$$
\begin{aligned}
& T_{1}(x)=\sum_{1}^{N}(y-u)^{2} \\
& T_{2}(x)=N p\left(\hat{b}-b_{0}\right)^{2} \quad \hat{b}=\left(U^{T} U\right)^{-1} U^{T} Y
\end{aligned}
$$

where $\hat{b}$ is the least square estimate of $b$, and $U=$ $\left[u\left(t_{0}\right) \ldots u\left(t_{+} N\right)\right]^{T}$. Note that in the test quantity $T_{2}(x)$, a normalization factor $N p=U^{T} U$ has been used, and $p$ is thus the mean power of $u$. The reason for this is to get a test quantity such that the significance level of the hypothesis tests becomes independent of the input signals.

The comparison study will be made by using the power function. It can be realized that $\sqrt{N p}\left(\hat{b}-b_{0}\right)$ is $N\left(0, \sigma_{v}\right)$ under $H_{0}$. This implies that $T_{2}(x) / \sigma_{v}^{2}$ is $\chi^{2}(1)$-distributed. Similarly it can be shown that $T_{1}(x) / \sigma^{2}$ is $\chi^{2}(N)$-distributed under $H_{0}$. The knowledge of these distributions can be used to find thresholds $J_{1}$ and $J_{2}$ such that a specific significance level, i.e. $\beta(\theta)$ for $\theta \in \Theta_{k}^{0}$, is obtained.

To evaluate the test quantities (6) and (7), two tests are constructed, based on $T_{1}(x)$ and $T_{2}(x)$ respectively. The standard deviation $\sigma_{v}$ is assumed to be 0.2 and then the thresholds are chosen such that the significance level for both tests becomes $\alpha=0.0034$. 


\subsection{Studying Power Functions}

We will compare the test quantities in three different cases: when fault mode $F_{b}$ is present, when fault mode $F_{\varphi}$ is present, and when fault mode $F_{b}$ is present and the test quantities are modified such that fault mode $F_{a}$ is decoupled.

3.1.1. Fault Mode $F_{b}$ Present This case corresponds to that the power functions for the case $a=a_{0}$, and $\varphi=\varphi_{0}$ are studied, i.e. along the $b$-axis of the fault state space $\Theta$. This means that the system model becomes linear and can be written as

$$
y(t)=b u+v
$$

Further, the power functions become functions of $b$, i.e. $\beta_{1}(b)$ and $\beta_{2}(b)$.
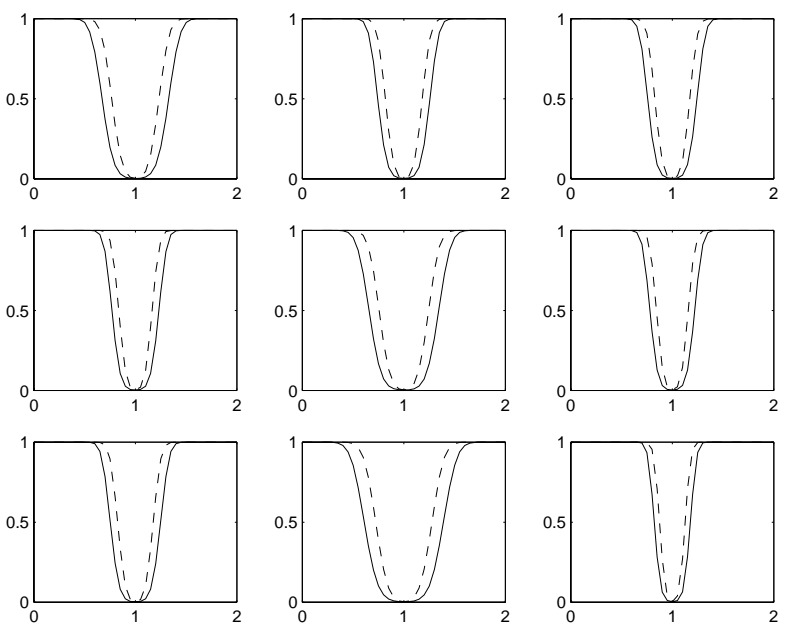

Fig. 1. The power functions $\beta_{1}(\theta)$ (solid) and $\beta_{2}(\theta)$ (dashed) for two tests based on $T_{1}(x)$ and $T_{2}(x)$. The result for 9 different input signals $u$ is shown.

The power functions $\beta_{1}(b)$ and $\beta_{2}(b)$ for 9 different input signals $u$, estimated by means of simulations, are plotted in Figure 1.

In the figure, it is seen that for all 9 different $u$ :s, the two power functions are equal for large deviations from $\theta_{0}$ but for other values, $\beta_{2}(\theta)$ (dashed) is greater than $\beta_{1}(\theta)$ (solid), i.e. $T_{2}(x)$ is better than $T_{1}(x)$. In other words, the estimate principle, with the estimated parameter the same as the one modeling the fault, here outperforms the prediction error principle.

3.1.2. Fault Mode $F_{\varphi}$ Present Now consider the fault mode $F_{\varphi}$, which means that $\varphi$ is a free variable while $b_{0}=1$ and $a_{0}=0$. The model (5) now becomes

$$
y(t)=|u|^{\varphi} \operatorname{sgn} u+v
$$

The two power functions $\beta_{k}(\varphi)$ for $T_{1}(x)$ and $T_{2}(x)$ respectively, are plotted in Figure 2. As before, 9 different input signals $u$ have been considered. In contrast to Figure 1, there are large differences between the different plots. This holds for both power functions $\beta_{1}(\varphi)$ and $\beta_{2}(\varphi)$. However, it is clear that $T_{2}(x)$ is very sensitive to different $u$ :s while $T_{1}(x)$ is more robust. Also, in all plots it no longer holds that $\beta_{2}(\varphi) \geq \beta_{1}(\varphi)$ for all $\varphi$. In most of the plots, $\beta_{1}(\varphi)$ is actually larger than $\beta_{2}(\varphi)$. It is obvious that the overall performance of $T_{1}(x)$ is much better than $T_{2}(x)$. Thus, in this case where the estimate principle uses an estimate of a parameter not modeling the fault, the prediction error principle outperforms the estimate principle.
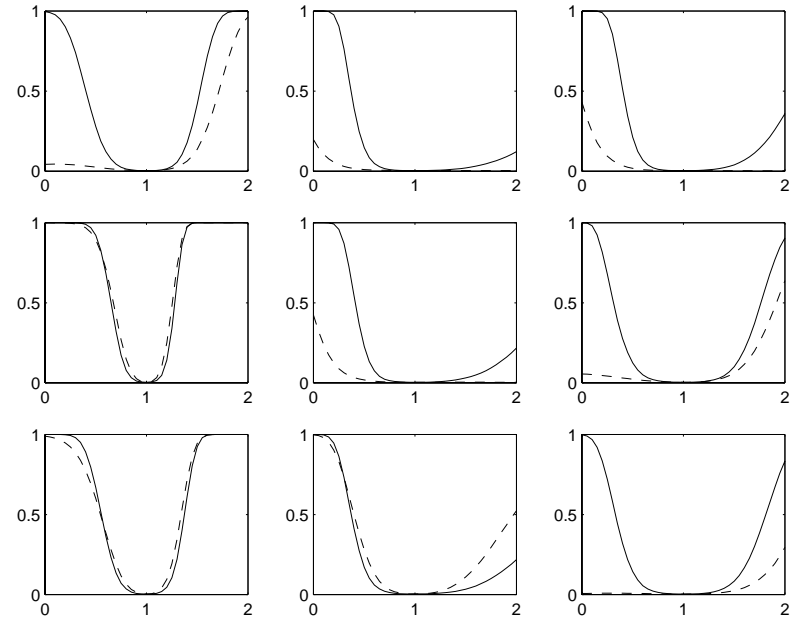

Fig. 2. The power functions $\beta_{1}(\varphi)$ (solid) and $\beta_{2}(\varphi)$ (dashed) for two tests based on $T_{1}(x)$ and $T_{2}(x)$.

The result for 9 different input signals $u$ is shown.

\subsubsection{Decoupling of $F_{a}$ and Fault Mode $F_{b}$ Present}

Next we will investigate how the test quantities $T_{1}(x)$ and $T_{2}(x)$ are affected by decoupling of the fault mode $F_{a}$. To do this, we construct two new test quantities $T_{1 a}(x)$ and $T_{2 a}(x)$ :

$$
\begin{aligned}
& T_{1 a}(x)=\min _{a} \sum_{t=1}^{N}(y(t)-\hat{y}(t \mid a))^{2}=\min _{a} \sum_{t=1}^{N}(y(t)-u(t)-a)^{2} \\
& T_{2 a}(x)=N p(\hat{b}-1)^{2} \quad \hat{b}=\underset{b}{\arg \min _{b, a}} \sum_{t=1}^{N}(y(t)-b u(t)-a)^{2}
\end{aligned}
$$

Note that all estimation problems involved are standard least square problems.

Tests using $T_{1 a}(x)$ and $T_{2 a}(x)$ are constructed with the significance level $\alpha=0.0034$ (the same as before). The parameter $a$ is chosen as $a=1$. The resulting power functions $\beta_{k}(b)$ corresponding $T_{1 a}(x)$, and $T_{2 a}(x)$ are then estimated via simulations. Included in the study are also $T_{1}(x)$ and $T_{2}(x)$, i.e. (6) and (7), but here with data compensated for the non-zero $a$, i.e. $y^{\prime}=$ $y-1$. The power functions for $T_{1 a}(x), T_{2 a}(x), T_{1}(x)$, and $T_{2}(x)$ are plotted in Figure 3. Here 4 different input signals $u$ have considered. We can see that the estimate principle also for this case, i.e. including decoupling, outperforms the prediction error principle. It should be remembered though, that the estimate principle implies that one extra parameter must be estimated, and this can in general be a substantial problem.

Also seen in the plots is that the dotted line is above the dashed, meaning that the test quantity $T_{2}(x)$ performs better than $T_{2 a}(x)$. However, this is the expected result since one less parameter has to be estimated using $T_{2}(x)$ compared to $T_{2 a}(x)$. Theoretically this can be explained by realizing that the variance of the least square estimate $\hat{b}$ in $T_{2 a}(x)$ is greater than the variance of $\hat{b}$ in $T_{2}(x)$.

\subsection{A Theoretical Study}

To find a theoretical motivation to why the estimate principle is better than the prediction error principle, we will here study a somewhat simplified case. Consider the model (8) but assume that $b \geq 0$ and the no fault case corresponds to $b=b_{0}=0$. We will consider two test quantities: $T_{1}(x)$ from $(6)$ and $T_{2}^{\prime \prime}(x)$ which we define as 

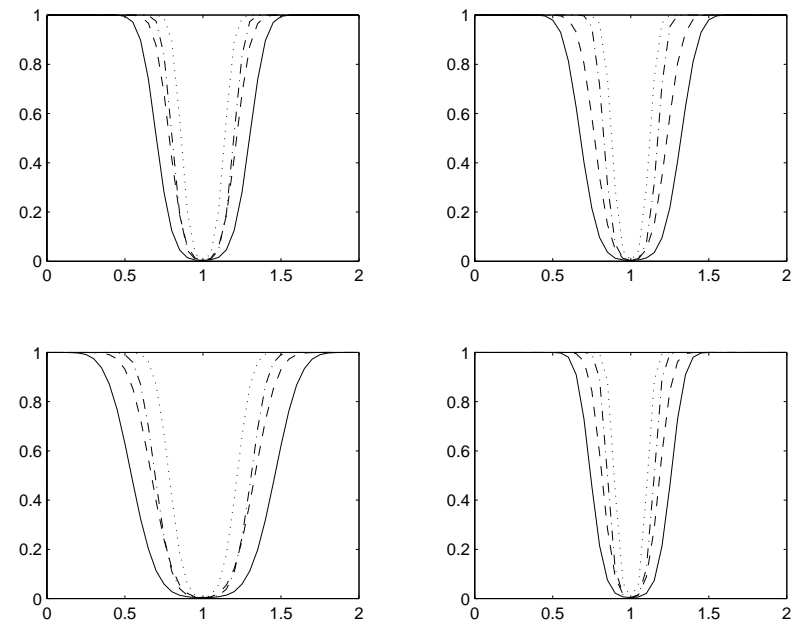

Fig. 3. The power functions $\beta_{1 a}(b)$ (solid), $\beta_{2 a}(b)$ (dashed), $\beta_{1}(b)$ (dash-dotted), and $\beta_{2}(b)$ (dotted) for tests based on $T_{1 a}(x), T_{2 a}(x), T_{1}(x)$, and $T_{2}(x)$ respectively. The result for 4 different input signals $u$ is shown.

$$
T_{2}^{\prime \prime}(x)=\sqrt{N p} \hat{b}=\sqrt{N p}\left(U^{T} U\right)^{-1} U^{T} Y
$$

Although not shown here, the power functions for corresponding tests are similar to the ones shown in Figure 1. That is, we can conclude that the test quantity based on the estimate principle, i.e. $T_{2}^{\prime \prime}(x)$, is better than the test quantity $T_{1}(x)$ based on the prediction principle.

Now consider the following theorem (Casella and Berger, 1990):

Theorem 1. If $f(\mathbf{x} \mid \theta)$ is the joint probability density function of $\mathbf{X}$, and $q(t \mid \theta)$ is the probability density function of $T(\mathbf{X})$, then $T(\mathbf{X})$ is a sufficient statistic for $\theta$ if, and only if, for every $\mathbf{x}$ in the sample space, the ratio $f(\mathbf{x} \mid \theta) / q(T(\mathbf{x}) \mid \theta)$ is constant as a function of $\theta$ (i.e. independent of $\theta)$.

With this theorem it can be shown that $T_{2}^{\prime \prime}(x)$, is a sufficient statistic for $b$. Next consider the following theorem (Casella and Berger, 1990):

Theorem 2. Consider testing $H_{0}: \theta \in \Theta_{0}$ versus $H_{1}$ : $\theta \in \Theta_{0}^{C}$. Suppose a test based on a sufficient statistic $T$ with rejection region $\mathcal{S}$, satisfies the following three conditions:

a. The test is a level $\alpha$ test.

b. There exists a $\theta_{0} \in \Theta_{0}$ such that $P\left(T \in \mathcal{S} \mid \theta_{0}\right)=$ $\alpha$.

c. Let $g(t \mid \theta)$ denote the probability density function of $T$. For the same $\theta_{0}$ as in (b), and for each $\theta^{\prime} \in \Theta_{0}^{C}$, there exists a $k^{\prime} \geq 0$ such that

$$
\begin{aligned}
& t \in \mathcal{S} \text { if } g\left(t \mid \theta^{\prime}\right)>k^{\prime} g\left(t \mid \theta_{0}\right) \quad \text { and } \\
& t \in \mathcal{S}^{C} \text { if } g\left(t \mid \theta^{\prime}\right)<k^{\prime} g\left(t \mid \theta_{0}\right)
\end{aligned}
$$

Then this test is a $\mathrm{UMP}^{1}$ level $\alpha$ test of $H_{0}$ versus $H_{1}$.

\footnotetext{
1 A test with power function $\beta(\theta)$ is a UMP (uniformly most powerful) level $\alpha$ test if there exist no other test with the same significance level $\alpha$ and with a power function $\beta^{\prime}(\theta)$ such that $\beta^{\prime}(\theta)>\beta^{\prime}(\theta)$ for any $\theta$.
}

All conditions (a), (b), and (c) can be easily shown to hold. Therefore we have the result that a hypothesis test based on $T_{2}^{\prime \prime}(x)$ is a UMP test. This means that there can not exist any test quantity better than $T_{2}^{\prime \prime}(x)$ for this hypothesis test.

\section{DIAGNOSING LEAKS IN THE AIR-PATH OF AN AUTOMOTIVE ENGINE}

In this section, diagnosis of leaks in the air-path of a turbo-charged gasoline engine (described in (Nyberg, $1999 a)$ ) is discussed. The air enters the engine and is compressed by the compressor. This results in a boost pressure higher than ambient pressure. The air then passes the throttle, enters the intake manifold, and finally leaves the manifold into the cylinders. Two types of leaks are considered: boost leakage before the throttle, and manifold leakage between the throttle and the cylinders. The model of the system is

$$
\begin{aligned}
m-k_{b} h_{b}\left(p_{b}\right) & =f\left(p_{b}, \alpha, p_{m}\right) \\
f\left(p_{b}, \alpha, p_{m}\right)+k_{m} h_{m}\left(p_{m}\right) & =g\left(p_{m}, n\right)
\end{aligned}
$$

where $m$ is the measured air mass-flow into the engine, $k_{b}$ is the effective area of the boost leakage, $p_{b}$ the measured boost pressure, $h_{b}\left(p_{b}\right)$ the flow function describing the air mass-flow through the boost leakage, $p_{m}$ the measured manifold pressure, $\alpha$ the meaasured throttle angle, $f\left(p_{b}, \alpha, p_{m}\right)$ the flow past the throttle, $k_{m}$ the effective area of the manifold leakage, $h_{m}\left(p_{m}\right)$ the flow function for the manifold leakage, $n$ the measured engine speed, and $g\left(p_{m}, n\right)$ the flow into the cylinders.

\subsection{Design of Test Quantities}

Three fault-modes are considered: No Fault $N F, k_{b}=$ $k_{m}=0$; Boost Leak $B L k_{b}>0, k_{m}=0$; and Manifold Leak $M L, k_{b}=0, k_{m}>0$. We assume that we want to design test quantities for the following two hypothesis tests:

$$
\begin{array}{ll}
H_{B L}^{0}: F_{p} \in M_{B L}=\{N F, B L\} & H_{B L}^{1}: F_{p} \in M_{B L}^{C}=\{M L\} \\
H_{M L}^{0}: F_{p} \in M_{M L}=\{N F, M L\} & H_{M L}^{1}: F_{p} \in M_{M L}^{C}=\{B L\}
\end{array}
$$

4.1.1. Prediction Principle As described in Section 2.2.1, the prediction principle is based on a comparison of signals and/or predictions of signals. It is straightforward to use this principle based on the model (9). Consider first the construction of the test quantity $T_{B L}^{p p}(x)$. (The index $p p$ denotes "prediction principle" to distinguish this test quantity from the one constructed in the next section.) The test quantity can in a first step be constructed in accordance with the formulas (4) and (3) as follows:

$$
\begin{aligned}
& T_{B L}^{p p^{\prime}}(x)= \min _{k_{b}} V_{B L}\left(k_{b}, x\right)= \\
&=\min _{k_{b}} \frac{1}{N} \sum_{t=1}^{N}\left(m-k_{b} h_{b}\left(p_{b}\right)-f\left(p_{b}, \alpha, p_{m}\right)\right)^{2}+ \\
& \quad+\frac{1}{N} \sum_{t=1}^{N}\left(f\left(p_{b}, \alpha, p_{m}\right)-g\left(p_{m}, n\right)\right)^{2}
\end{aligned}
$$

To save space, the time-argument of all variables have been skipped. The expression (10) consists of two terms. Ideally, the first of these terms will always be zero for all possible fault modes. However, in reality the first term is non-zero and acts as an unknown disturbance in the test quantity $T_{B L}^{p p}(x)$. Since the 
first term only acts as a disturbance, it can be skipped which results in the test quantity

$$
T_{B L}^{p p}(x)=\frac{1}{N} \sum_{t=1}^{N}\left(f\left(p_{b}, \alpha, p_{m}\right)-g\left(p_{m}, n\right)\right)^{2}
$$

Similarly, the test quantity $T_{B L}(x)$ is constructed as

$$
T_{M L}^{p p}(x)=\frac{1}{N} \sum_{t=1}^{N}\left(m-f\left(p_{b}, \alpha, p_{m}\right)\right)^{2}
$$

Also here we have skipped the term that is close to zero all the time. The only drawback with this approach, i.e. to skip one of the terms, is when an unpredicted fault occurs, i.e. a fault not belonging to any of the fault modes $B L$ or $M L$. Then it can happen that this fault is mistaken to belong to $B L$ or $M L$.

In conclusion, the test quantity $T_{B L}^{p p}(x)$ has been constructed so that the fault modes $B L$ and $N F$ are decoupled, and $T_{M L}^{p p}(x)$ has been constructed so that the fault modes $M L$ and $N F$ are decoupled. This fulfills the requirements of the two hypothesis tests specified above.

4.1.2. Estimate Principle Using the estimate principle in accordance with Section 2.2.2, we base our test quantities on estimates of the effective areas $k_{b}$ and $k_{m}$. First we discuss the construction of the test quantity $T_{M L}^{e p}(x)$. The non-normalized version becomes:

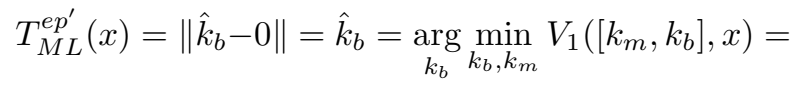

$$
\begin{aligned}
& =\underset{k_{b}}{\arg } \min _{k_{b}, k_{m}} \frac{1}{N} \sum_{t=1}^{N}\left(m-k_{b} h_{b}\left(p_{b}\right)-f\left(p_{b}, \alpha, p_{m}\right)\right)^{2}+ \\
& +\frac{1}{N} \sum_{t=1}^{N}\left(f\left(p_{b}, \alpha, p_{m}\right)-g\left(p_{m}, n\right)+k_{m} h_{m}\left(p_{m}\right)\right)^{2}={ }^{*} \\
& ={ }^{*} \arg \min _{k_{b}} \frac{1}{N} \sum_{t=1}^{N}\left(m-k_{b} h_{b}\left(p_{b}\right)-f\left(p_{b}, \alpha, p_{m}\right)\right)^{2}= \\
& =\arg \min _{k_{b}} V_{2}\left(k_{b}, x\right)
\end{aligned}
$$

Note that the measure $\|\cdot\|$ is here defined as the identity function. The function $V_{1}\left(\left[k_{m}, k_{b}\right], x\right)$ is a model validity measure. It is here trivially derived in analogy with $T_{B L}(x)$ and $T_{M L}(x)$ (which are also model validity measures) from the previous section. The equality marked $={ }^{*}$ follows from the fact that the coefficient $k_{b}$ is only present in one of the terms of $V_{1}\left(\left[k_{m}, k_{b}\right], x\right)$. The minimization of $V_{2}\left(k_{b}, x\right)$ is a linear regression problem. Therefore the least-square technique was used for both $T_{M L}^{e p^{\prime}}$ and $T_{B L}^{e p^{\prime}}$.

With normalization, to make the significance level of the hypothesis tests independent of the input signals, the test quantity $T_{M L}^{e p}(x)$ become

$$
T_{B L}^{e p}(x)=\sqrt{\varphi_{m}^{T} \varphi_{m}} T_{B L}^{e p^{\prime}}(x)=\sqrt{\varphi_{m}^{T} \varphi_{m}} \hat{k}_{m}
$$

where $\varphi_{m}^{T}=\left[h_{m}\left(p_{m}\left(t_{1}\right)\right) \ldots h_{m}\left(p_{m}\left(t_{N}\right)\right)\right]$. The test quantity $T_{M L}^{e p}(x)$ is defined similarly. With these two test quantities, decoupling has been achieved in accordance with the specifications of the two hypothesis tests.

\subsection{A Comparison Between the Prediction Principle and the Estimate Principle}

The diagnosis problem investigated here is in principle the same as the one investigated in Section 3.2. There we saw that the estimate principle gives the best possible test quantity. This means that the test quantities $T_{B L}^{e p}(x)$ and $T_{M L}^{e p}(x)$ given in (13) should be better than $T_{B L}^{p p}(x)$ and $T_{M L}^{p p}(x)$ given in (11) and (12) respectively.

To make the comparison, we need to obtain the power function for tests corresponding to all four test quantities. In this situation, where there is no knowledge or assumptions about the model errors or the measurement errors, measurements on the real process must be used. Only a limited number of leakage areas are studied, corresponding to $0,1,2$, and $3.5 \mathrm{~mm}$ diameter. To estimate the probability density function in this case is difficult because of the large amount of data that would be needed. Only 24 independent data sets were used for the analyses and therefore a simpler and less accurate approach has to be chosen.

Both boost leakage and manifold leakage were studied, but because of space limitations, only the results when a manifold leakage was present, are presented here. The complete study can be found in (Nyberg, $1999 b)$. The results of the study, for manifold leakage present, are shown in Figures 4 to 5. Consider first Figure 4 . The $\mathrm{x}$-axis represents the different leakage areas corresponding to $0,1,2$, and $3.5 \mathrm{~mm}$ diameter. For each leakage area, the test quantities $T_{B L}^{e p}(x)$ and $T_{M L}^{e p}(x)$ were calculated for each of the 24 data sets. The values of $T_{B L}^{e p}(x)$ and $T_{M L}^{e p}(x)$ are indicated with " $\mathrm{x}$ " and "O" respectively. To make the plot more clear, all " $\mathrm{x}$ ":s have been moved slightly to the right. For each leakage area, also the mean and the standard deviation are calculated and shown as horizontal bars. The middle bar is the mean and the upper and lower bars are two times the standard deviation.

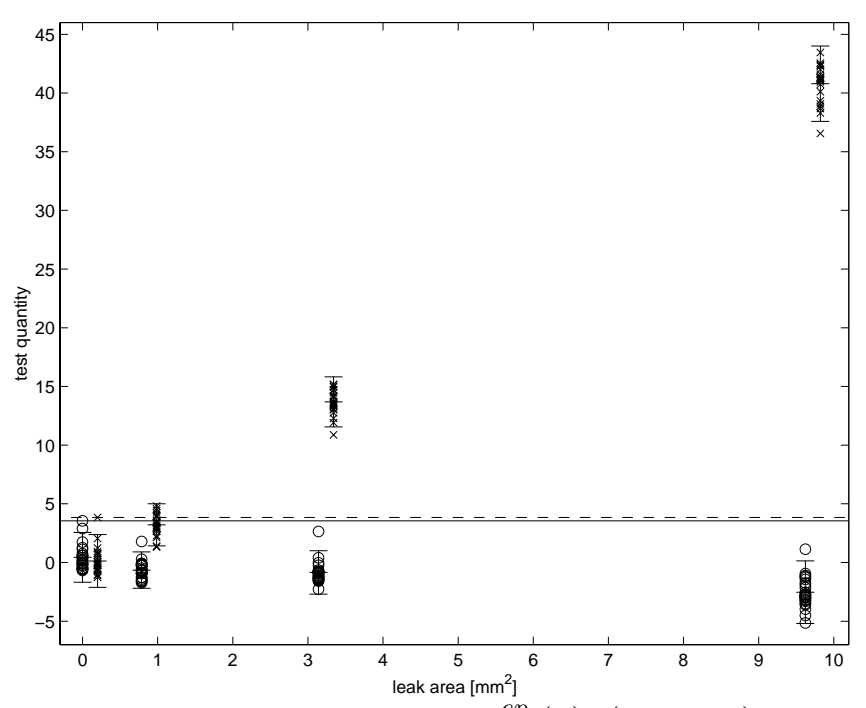

Fig. 4. The test quantities $T_{B L}^{e p}(x)$ (x-marks) and $T_{M L}^{e p}(x)$ (circles), based on the estimate principle, for different manifold-leakage areas.

To be able to make a comparison, thresholds need to be chosen such that the significance levels in two compared hypothesis tests become equal. Since we don't have the probability density function, this can 


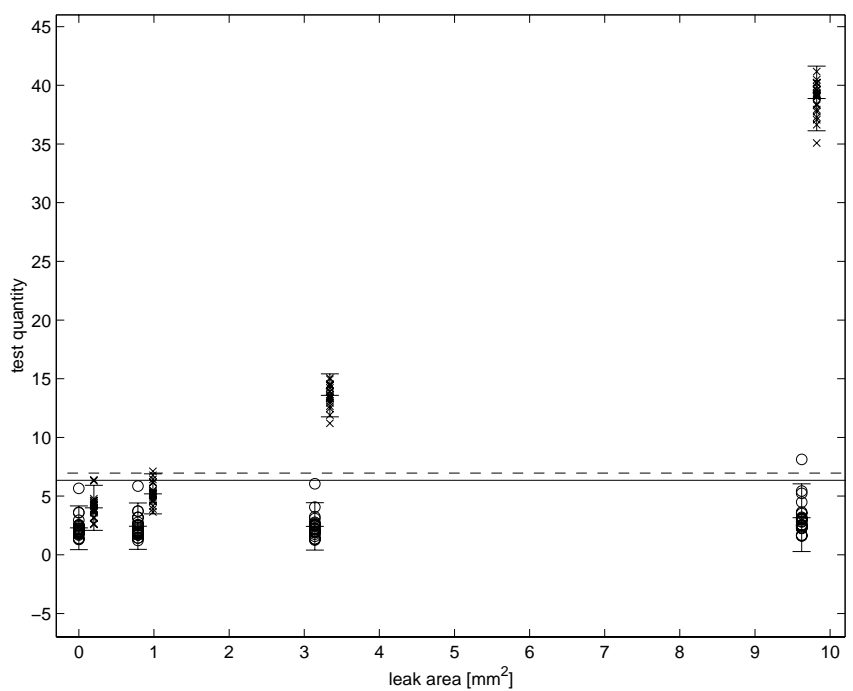

Fig. 5. The test quantities $T_{B L}^{p p}(x)$ (x-marks) and $T_{M L}^{p p}(x)$ (circles), based on the prediction principle, for different manifold-leakage areas.

not achieved. Instead, each threshold is chosen as the maximum value of the corresponding calculated test quantity in the fault-free case. Consider again Figure 4. The maximum values of the test quantities $T_{B L}^{e p}(x)$ and $T_{M L}^{e p}(x)$ for the fault-free case, i.e. leakage area 0 , are marked by the dashed and solid lines respectively.

The power function is the probability to reject $H_{0}$, i.e. the probability that the test quantity is above the threshold. As was said above, we don't have the probability density function, which means that exact values of the power function can not be calculated. However, by studying Figure 4 and looking at the mean and standard deviation values, we can quite easily get a coarse estimate of the probability that the test quantity is above the threshold. For example, it is obvious that the power function $\beta_{B L}^{e p}\left(\left[\begin{array}{ll}0 & k_{m}\end{array}\right]\right)$, corresponding to $T_{B L}^{e p}(x)$, will increase as the leakage area increases. Also, we can conclude that the power function for the leakage with an area of $3.1 \mathrm{~mm}^{2}$ is large, which means that it should be no problem to detect a manifold leakage with this area. Further, the power function for the leakage with an area of 0.8 $\mathrm{mm}^{2}$, is probably quite low, which means that it is hard to distinguish this leakage from the no-leakage case.

Now return to the comparison of test quantities. First compare Figure 4, showing the test quantities $T_{B L}^{e p}(x)$ and $T_{M L}^{e p}(x)$, and Figure 5 , showing the test quantities $T_{B L}^{e p}(x)$ and $T_{M L}^{e p}(x)$. We see that the test quantity $T_{B L}^{e p}(x)$ is slightly more above the threshold than $T_{B L}^{p p}(x)$. This means that the power function $\beta_{B L}^{e p}\left(\left[\begin{array}{ll}0 & k_{m}\end{array}\right]\right)$ is very likely to be larger than $\beta_{B L}^{p p}\left(\left[\begin{array}{ll}0 & k_{m}\end{array}\right]\right)$. In other words, for the manifold leakage, the estimate principle is better than the prediction principle. Although not shown here, the same result was obtained also for boost leakage.

\section{CONCLUSIONS}

In the theoretical investigation in Section 3, the focus was mainly on specific examples. In spite of this, we are able to summarize the following conclusions:
- Test quantities based on estimates can have very good performance for the fault mode corresponding to the estimated parameter.

- For other fault modes, the performance might be quite bad and also highly dependent on the input signal.

- Decoupling degrades the performance of both the prediction principle and the estimate principle but the relation that the estimate principle is better than the prediction error principle still holds.

The practical study in Section 4 focus on the first of these three issues. Even though it was not possible to estimate the density functions, it could be concluded that, of the two principles studied, the best principle for diagnosing leakage is the estimate principle. In the theoretical study in Section 3.2, we used the assumption of independently and identically Gaussian distributed noise. This assumption do not hold in the real case investigated in this section, but nevertheless it is obvious that the conclusion that the estimate principle is better than the prediction principle, still holds. According to the third issue above, this conclusion should hold also if some other faults need to be decoupled, e.g. a bias fault in the throttle-angle sensor.

The practical relevance of the second issue above, is that if also other faults are diagnosed by means of the same test quantities, it is no longer sure that the estimate principle should be the first choice.

In production cars, a principle similar to the prediction principle is often used, e.g. see (Air Leakage Detector for IC Engine, 1994). A reason for this may be that models of the leaks are not required (see the test quantities described by (11) and (12)). It is interesting to note that the technique to use models of the leaks and then estimate the leakage area, performs better than the solution common in production cars. This method was developed in (Nyberg and Perkovic, 1998) and is patent pending by SAAB Automobile.

\section{REFERENCES}

Air Leakage Detector for IC Engine (1994). Patent $R D 368014$.

Casella, G. and R.L. Berger (1990). Statistical Inference. Duxbury Press.

Isermann, R. (1993). Fault diagnosis of machines via parameter estimation and knowledge processing tutorial paper. Automatica 29(4), 815-835.

Nyberg, M. (1999a). Model based diagnosis of both sensor-faults and leakage in the air-intake system of an SI-engine. SAE Paper 1999-01-0860.

Nyberg, M. (2000). Model based fault diagnosis using structured hypothesis tests. Fault Detection, Supervision and Safety for Technical Processes. IFAC. Budapest, Hungary.

Nyberg, M. and A. Perkovic (1998). Model based diagnosis of leaks in the air-intake system of an SI-engine. SAE Paper 980514.

Nyberg, Mattias (1999b). Model Based Fault Diagnosis: Methods, Theory, and Automotive Engine Applications. PhD thesis. Linköping University. URL: http://www.fs.isy.liu.se/Publications/. 